\title{
Comprensiones narrativas de los factores asociados al desempeño académico en estudiantes de Boyacá, Colombia*
}

\section{Narrative understanding of factors associated with academic performance in students of Boyacá, Colombia}

\author{
Jenny Paola Burgos Díaz* \\ ORCID 0000-0003-3087-6460 \\ Universidad de Boyacá, Colombia
}

Recibido: 20 de marzo de 2017 Revisado: 13 de abril de 2017 Aceptado: 3 de junio de 2017

\section{Resumen}

El presente artículo es un estudio cualitativo de tipo narrativo cuyo objetivo principal fue comprender la relación entre los factores psicosocial, familiar e institucional, y el desempeño académico. Participaron de manera voluntaria 16 estudiantes en situación de prueba académica en dos escenarios conversacionales. Para el procesamiento de la información se diseñó una matriz de análisis que permitió categorizar las narrativas en función de los dóminos temáticos de la investigación. Los análisis de las narrativas presentadas por los estudiantes en los escenarios realizados permitieron comprender el significado de relevancia que los estudiantes le dan a la relación con el docente; la importancia del apoyo familiar como un elemento motivador; las dificultades familiares como un interferente; la influencia de los pares en la toma de decisiones, mayor interés por las actividades sociales que sobre aspectos académicos y finalmente características personales de afrontamiento evasivo con cierta regularidad frente a las dificultades académicas reportadas.

Artículo de investigación. http://dx.doi.org/10.15332/s1794-9998.2018.0001.10

Correspondencia: Jenny Paola Burgos, Facultad de Psicología, Universidad de Boyacá. Dirección postal: carrera 2E No. 64-169, Tunja, Boyacá. Colombia. Correo electrónico: jepaoburgos@uniboyaca.edu.co. 
Palabras clave: narrativas, factores: psicosocial, familiar e institucional, desempeño académico.

\begin{abstract}
This article presents a qualitative study belonging to a narrative type, whose main objective was to understand the relationship between psychosocial, family and institutional factors and academic performance. 16 students undergoing academic trial participated voluntarily in two conversational scenarios. An analysis matrix was designed to carry out data processing through categorization of narratives according to the thematic domains of the research. The analysis of the students' narratives at the implemented scenarios allowed the comprehension of the meaning of relevance that students attribute to their relationship with teachers; the importance of family support as a motivational element and family difficulties as an interference; the influence of their peers in decision making and greater interest in social activities than academic aspects, and finally, frequent personal features of evasive coping, facing reported academic difficulties.
\end{abstract}

Keywords: Narratives, Psychosocial, family and institutional factors, Academic performance.

\section{Introducción}

El desempeño académico de los estudiantes es un tema de interés, en el que se ha logrado reconocer, mediante diversos estudios de investigación, la incidencia de diferentes factores, como claves para comprender la complejidad de lo que implica el aprendizaje, la evaluación y finalmente el desempeño académico. Es pues el desempeño académico un reflejo de procesos complejos que implican elementos personales, sociales, académicos y familiares, que finalmente redundan en una adquisición de conocimiento que permite ser reflejado en un resultado evaluativo, así mismo en un proceso constante de transformación y aprendizaje que se da a lo largo de toda la experiencia universitaria (Urquijo, 2002).

De acuerdo con lo anterior, la presente investigación pretendió abordar esta temática, como una forma de dar respuesta a la dificultad que enfrentan los estudiantes que se encuentran en una situación de bajo desempeño académico y ante la posibilidad de caer en deserción universitaria.

Desde el reconocimiento de la complejidad del fenómeno se buscó comprender la relación entre los factores: psicosocial, familiar e institucional, y el desempeño académico. Este último entendi- do como el proceso de formación integral y académico que viven los estudiantes de educación superior. Desde una metodología cualitativa, principalmente en la comprensión de las narrativas de los estudiantes como orientadores de sus propias vivencias, así mismo como agente que favorece y evidencia el cambio, que permitieron acceder a su realidad a través de las narrativas, encontrándose que no solo son conscientes de sus dificultades de bajo desempeño académico, sino que también han logrado relacionar esta situación con aspectos como: la relación con el docente, la influencia de los amigos y la familia, además de reconocer las características personales que los han llevado a comportarse de manera diferente frente al problema académico que presentan.

\section{Relación entre el desempeño académico y los factores: familia, institucional y psicosociales de estudiantes universitarios}

\section{Desempeño académico}

La transición de la educación básica secundaria a superior trae consigo una serie de cambios en 
los estilos de vida de los bachilleres, lo cual plantea retos para la adaptación tanto de los jóvenes como de sus familias en sus procesos emocionales, relacionales, sociales y académicos. Así mismo requiere que la institución universitaria, esté en constante actualización para dar respuesta a estos retos. Como afirma Soares, Almeida y Guisande (2011) y Berger et al. (2013) es una confrontación con aspectos emocionales, sociales, académicos e institucionales, lo que a su vez genera en los estudiantes tensiones que en ocasiones no se logran resolver, lo que da como resultado un bajo desempeño académico.

En el ámbito académico, se tienen en cuenta tanto a los estudiantes como los actores que componen la institución educativa como directivas y docentes, que evidencia una interacción donde se ponen en juego la adquisición del conocimiento y su respuesta valorativa, además de la complejidad de los procesos formativos vistos desde una perspectiva integral. En este sentido, es necesario reconocer que dentro de la revisión desarrollada no se encontró un concepto específico sobre desempeño académico, lo más cercano al concepto fueron definiciones alrededor del rendimiento académico, sin embargo se considera que la definición presentada por Montes y Lerner (2011) es la que se aproxima al propósito del presente estudio, quienes la entienden como "la manera continua de lograr conocimiento, el cual siempre será susceptible de ser ampliado, revisado, rebatido y de constituirse en objeto de nuevas interpretaciones." (p. 15). Definición que favorece el objetivo de la educación que está orientada en fomentar nuevas formas de ver, interpretar y construir el mundo.

\section{Factor familiar}

De acuerdo con Minuchin y Fishman (2004) la concepción de familia da cuenta de una organización social que comparte estructuras, comportamientos, y en sí mismos, logran construir una identidad que los caracteriza, además de enfrentarse a una serie de tareas de desarrollo, siendo estas tareas las que de alguna forma orientan los patrones de comportamiento, así como las representaciones del mundo. En este sentido, el impacto que tiene la familia en cada uno de sus miembros es determi- nante para la experiencia subjetiva de las diversas tareas que se pueden realizar a lo largo de la vida.

Así mismo refieren Minuchin y Fishman (2004) que la familia determina las respuestas de sus miembros mediante estímulos que se derivan de sus dinámicas propias y de la influencia del entorno, siendo el grupo familiar un evaluador de los comportamientos y las experiencias de sus miembros, por tanto, direccionan o limitan las prospectivas y las acciones para la consecución de metas.

En este punto y retomando el propósito de la investigación, la familia influye directamente en las decisiones vocacionales y los resultados académicos que presente el estudiante, puesto que se ponen en juego los esfuerzos de los padres para apoyar al hijo en la consecución de sus metas, además de las expectativas de todos los miembros de la familia sobre su desarrollo y realización, aspecto que cobra sentido en las narrativas de los estudiantes como un elemento motivador. Esto se logra articular con lo planteado por Guevara et al (2013); quienes lograron identificar que existen implicaciones entre el nivel educativo del padre y las expectativas del estudiante, lo que puede entenderse si se considera que en la estructuración de la familia y el manejo de autoridad y roles, se encuentra que un gran porcentaje vive en familias donde existe claridad en estos aspectos, por lo que la figura paterna mantiene un significado de influencia y representatividad importante en el sistema familiar.

\section{Factor institucional}

Lo institucional se entiende como el contexto universitario, en el cual el estudiante adelanta su proceso de formación profesional, razón por la cual se tienen en cuenta lineamientos y políticas, modelo pedagógico, incentivos, servicios, cultura corporativa, elementos misionales, visiónales, organigrama, personal directivo, administrativos y docentes, entre otros aspectos que permitan conocer la realidad de la institución educativa universitaria, ya que se reconoce la influencia de este entorno en el desempeño académico del estudiante, como lo evidencia la revisión empírica sobre el tema. 
La interacción entre los actores que participan en contextos de formación superior permite lograr, según Jornet (2012) una comunicación asertiva entre docentes y estudiantes, lo cual contribuye a la libre expresión de las emociones. Por su parte, Saccsa (2010) concluye que el buen trato entre profesores tutores y estudiantes asegura un buen clima universitario, desde los directivos hasta los cargos subordinados. También Reparaz, Touron y Villanueva (1990) encontraron que los eventos culturales y el hacer partícipes a los estudiantes de las actividades extracurriculares promueve el sentido de pertenencia con la institución.

Respecto a la praxis del conocimiento en la educación superior, Chica, Galvis y Ramírez (2009) señalan que los estudiantes de jornada completa, tienden a tener un nivel académico por encima de los estudiantes que reciben sus clases fines de semana o en horas de la noche, lo cual denota que el horario, quizá el tiempo y el estar en continuo contacto con la institución afectan el rendimiento académico en los estudiantes.

\section{Factor psicosocial}

Este factor tiene en cuenta las características de personalidad del estudiante y la influencia del entorno social en relación a su desempeño académico, como bien lo refiere Guzmán y Pacheco (2014), quienes señalan que existe una serie de factores asociados al fracaso o éxito escolar, que van desde lo personal hasta lo sociocultural; es decir, una mezcla de circunstancias individuales y sociales que trascienden y aplican en la educación superior y en el contexto, lo cual evidencia la complejidad del rendimiento académico en relación con factores internos y externos que participan. De igual manera, Rosenberg, Schooler y Schoenbac (1995), citados en Pérez y Urquijo (2001), sostienen que características personales como la autoestima académica influyen en el rendimiento escolar, así como Luthar y Blatt (1995), quienes señalan que los sujetos con alto grado de autocrítica muestran mayor depresión, situación que se asocia con dificultades en el rendimiento académico y el funcionamiento interpersonal. De igual modo, Reinherz, Frost y Pakiz (1991) afirman que los adolescentes con altos valores de depre- sión reportan mayor número de problemas emocionales y de conducta, logro académico inferior y mayor abuso de drogas. Lo anterior permite identificar como el reconocimiento de los recursos en el joven le permite tener mayores herramientas a la hora de enfrentar las demandas del contexto universitario desde lo personal, lo académico y lo social, aspectos que guardan relación sobre los resultados alcanzados por cada estudiante en su proceso formativo.

\section{Método}

\section{Diseño}

Este estudio se abordó desde la narrativa conversacional, en la cual los participantes producen e interpretan sus propios relatos de la vida cotidiana. A su vez, el investigador/interventor también requiere comprender el modo de participar en el proceso conversacional, en tanto puede disponerse a ser testigo, narrador o co-narrador. De igual manera, la investigación se basó en postulados emergentes de la propuesta de la cibernética de segundo orden y de la complejidad, que permiten organizar escenarios comprensivos e interventivos a partir de la construcción de guiones a la luz de los focos de análisis de la presente investigación, con el fin, no solo de animar la conversación, sino también de transformar realidades hacia el recurso y la posibilidad. La metodología cualitativa empleada pretendió orientar y generar procesos de comprensión a los fenómenos humanos y sociales a partir de la interpretación y el análisis de narrativas desde una perspectiva reflexiva y hermenéutica, con lo que se busca generar procesos comprensivos desde el concepto de las narrativas y la organización de la realidad, con el lenguaje como organizador y coordinador del comportamiento humano (Echeverría, 2003).

\section{Participantes}

Participaron 16 estudiantes matriculados en la Universidad de Boyacá sede Sogamoso, entre los 17 y 27 años. En su mayoría se encontraban entre segundo y séptimo semestre de los programas de 
Derecho, Psicología, Administración, Ingeniería Ambiental y Arquitectura, todos manifestaron su voluntad de participar ante la invitación pública que se realizó al interior de la Universidad.

\section{Procedimiento}

Los escenarios conversacionales fueron grabados y transcritos, para posteriormente ser analizados mediante una matriz que se diseñó para tal fin, la cual contrasta el desempeño académico con los factores (institucionales, familiares y psicosociales) y las narrativas (historia, memoria y acontecimiento). La narrativa de historia se entienden como los relatos saturados del problema, la memoria por su parte deja ver algo de recurso y el acontecimiento evidencia la re-significación del problema hacia una mirada de cambio y mejora, lo cual permite dar cuenta del trabajo interventivo realizado en los escenarios conversacionales.

\section{Resultados}

\section{Historia - psicosocial}

Este apartado relaciona las historias, es decir las narrativas dadas por el estudiante, las cuales dan cuenta de versiones saturadas del problema (en este caso dificultades académicas) relacionándolas con elementos personales y sociales de su vida.

Las historias de los participantes están organizadas a partir de la representación de la "farra" como un evento que genera dificultad para su proceso académico, sin presentar alternativas para su solución, como lo muestra la siguiente narrativa:

E8: No porque hay el amigo que es farrero, entonces dice 'vamos no entre y vamos mejor alli' y no aggg, pues bueno, listo. (Esc.1)

Por otro lado, se presentan narraciones de los estudiantes, relacionadas con las asignaturas cursadas, los intereses y el desempeño que se tiene

$1 \quad$ Farra en este contexto universitario se refiere a espacios de diversión que tienen los estudiantes donde generalmente socializan con otros, se consume licor y se baila diferentes géneros musicales. al interior de estas, atribuyendo que la falta de interés e inasistencia a clase y el no cumplimiento de las tareas se debe al tema que trata la asignatura y en otras ocasiones a la actitud del docente.

Así mismo las historias dan cuenta de las consecuencias del problema de estar atrasado académicamente, pues eso influye en lo social, porque ya no se ven con los mismos compañeros y es necesario formar nuevos vínculos que permitan realizar los trabajos en grupo, los cuales al parecer no siempre resultan bien. Lo anterior evidencia como la interacción social entre compañeros guarda una relación con el desempeño académico, en este sentido, tener una consecuencia social por no estar en línea puede generar sentimientos de ser diferente, y esto se siente aún más visible cuando hacen comparaciones con los pares que van en línea, como lo muestra la siguiente narrativa:

P1: Eso es muy frustrante, yo siento frustración porque en algunas materias casi la mayoría yo ya no me veo con mis amigos con los que inicie, entonces es eso, o digamos para volver a coger confianza con alguien de que un trabajo o algo así, entonces es como eso, que baja mucho el ánimo. (Esc. 2: R:26).

\section{Memoria - psicosocial}

Las memorias, entendidas como relatos de los participantes que denotan algo de recurso en sus vivencias, están vinculadas a la posibilidad de reconocerse en facetas donde han logrado comportarse de forma aplicada, responsable, motivada al cumplimiento del logro, situación que muestra la posibilidad de cambio y mejora ante dificultades que puedan enfrentar en el entorno académico. Si bien, reconocen que tiene el potencial y el recurso para que esto sea posible, aun sus comprensiones y no se visualiza en sus acciones, como se puede evidenciar en la siguiente narrativa:

E3: (...) entonces pienso que influye mucho el manejo del tiempo, qué hago con mi tiempo, en qué lo gasto, el tema de los hábitos también, qué tipo de hábitos formo, qué tipo de hábitos quiero cambiar por unos positivos, aparte de eso yo pienso que tanto los ßhábitos 
como la organización influyen también en la actitud y la ganas que uno le pone a eso. (Esc.1)

En este relato, el estudiante evidencia un reconocimiento de estrategias que pueden serle útiles para mejorar en su desempeño, lo que le permite encontrar en el manejo del tiempo un elemento importante, que debe ser ajustado para que el cambio sea posible. Esto evidencia que el afrontamiento evitativo fue una de las causas por las cuales se mantuvo la pérdida de la misma materia por tres semestres consecutivos. El reconocimiento del error aparece como una oportunidad de cambio ante la posibilidad de pensarlo de una forma distinta a futuro.

De esta forma se presentan relatos que se configuran como alternativos porque dejan ver posturas de corresponsabilidad, donde se reconoce que factores externos como lo social e institucional juegan un papel importante, y también se observan relatos de autonomía y libertad sobre la posibilidad de reescribir su presente y futuro, como lo evidencia la siguiente narrativa:

P1: pues que efectivamente hay más salidas, para salir de esta situación, para o sea, para obtener como esa, sí, como esa situación y verla como que se puede solucionar, que tenemos más opciones y que podemos salir adelante, igual es cuestión de tomar conciencia y de darse cuenta de que estamos haciendo algo mal efectivamente. (Esc.2: R: 18)

Por otra parte, se presentan narrativas que dan cuenta del tránsito del colegio a la Universidad y de las diferencias que esto suscita, viéndose en el colegio una experiencia de aprendizaje movilizado por el castigo y la recompensa versus un escenario que se conecta directamente con una elección vocacional y unas dinámicas de aprendizaje que requieren del ejercicio de autonomía para tener éxito. El aumento de las responsabilidades pone en juego las estrategias de afrontamiento y la tolerancia a la frustración, en donde, aunque no todo lo que debe hacerse resulta placentero, pero se asume, porque se entiende como parte de las responsabilidades que debe asumir el estudiante, como parte de su ciclo vital.
Por otro lado, se evidencia como mantenimiento del problema una actitud facilista, fruto de la historia escolar vivida, también se observó como una comprensión importante da cuenta de la necesidad de implementar nuevas estrategias acordes con su realidad presente.

\section{Acontecimiento - psicosocial}

En cuanto a los acontecimientos, se logra visibilizar que los estudiantes comienzan a narrar algunos hábitos como el manejo y organización del tiempo tienen un impacto positivo en su desempeño, así mismo reconocen la asistencia a clase como un factor fundamental para su desempeño, por lo que se ve implicado el compromiso y la continuidad de los procesos.

P3: Lo que decía (...) de las prioridades, yo creo que en este momento al estar en prueba académica y ya pues la primera reunión que tuvimos, muchos de los que estamos acá empezamos a darle prioridades a las cosas, prioridades a determinadas actividades que tenemos y bien o mal pues ahorita digamos con todas las materias que estamos viendo (...) seguir adelante y seguir bien con las materias que estamos viendo (...) (E2:R:42)

Así mismo, los relatos dan cuenta de un estudiante que también identificó problemas en su rendimiento académico y buscó estrategias como interesarse en el tema, que para él le ha funcionado. Elementos que se organizan desde una reflexión de autonomía, de entender la importancia de esforzarse por el cumplimiento de sus metas, lo que finalmente puede llegar a favorecer el rendimiento académico.

Finalmente los estudiantes dan cuenta del sentido que tiene para ellos encontrarse en este proyecto académico, donde resulta inspirador, no solo la satisfacción personal ante el cumplimiento de las metas, sino también el beneficio para la familia con este progreso, así mismo reconoce que la experiencia de aprendizaje en la universidad ha impactado su proceso de búsqueda de identidad, que se evidencia en cambios narrados como positivos, que dan cuenta de un progreso 
personal y académico, como se indica en el siguiente relato:

P1: Yo de diez palabras decía nueve groserías y el estar en la universidad me ha ayudado con respecto a eso en lo que uno se relaciona en otras situaciones, en otro aspectos y debe tener una actitud diferente, ya no está con los amigos del barrio, con la recocha, con la grosería, sino aprende uno a diferenciar ciertos contextos en los cuales usted debe actuar de una manera adecuada, creo que para mí eso ha sido primordial, principalmente en la forma como ahora actuó, como ahora pues no se me deja de salir el llanero pero sí, en ese proceso de aprendizaje estoy. (R153)

\section{Historia - familiar}

Las historias de los participantes en cuanto a la familia están centradas en la presión que ejerce sobre la elección de la carrera, se hace notorio en los relatos, que en ocasiones la familia tiene una tradición en la formación y se espera que ellos den continuidad a esta línea. Como se expresa en el siguiente relato.

Bueno para la elección de la carrera en mi familia todos son ingenieros, entonces tenía como seguirla (...), no quería estudiar ingeniería ambiental, quería estudiar ingeniería de petróleos, pero no se pudo (...) (E1, Esc1)

Así mismo, los relatos se organizan desde el reconocimiento que hacen los estudiantes del problema de desempeño académico relacionado con problemáticas familiares, que lleva a que el estudiante desconozca los límites a sus diferentes situaciones de su vida, lo que permite que las dificultades en la familia interfieran con los procesos académicos, como se muestra a continuación:

Bueno, en mi caso tuve una serie de problemas con una niña de la universidad de acá, jeh! se metió con mi papá y eso me bajonió mucho, entonces no quería venir a estudiar, y pues la universidad cero para mí, porque la tenía que ver constantemente, entonces él como que la prefirió a ella y no a mí (...) (E2, Esc1).
En este sentido se observa que existe una dificultad en cuanto a los procesos de diferenciación que se presentan en las diversas experiencias familiares y académicas, lo que evidencia el impacto que tiene la familia en el desempeño académico.

\section{Memorias - familia}

En cuanto a lo familiar, se presentan memorias en los estudiantes en la medida en que la familia se organiza como posibilidad, siendo un factor importante en su desempeño, como un elemento posibilitador de apoyo, respaldo y motivación extrínseca, lo que les permite proyectarse a futuro. El estudiante narra, que a pesar de que exista una tradición en la formación, él ha logrado manifestar sus intereses, y esto le ha permitido estudiar lo que le gusta.

Así mismo se observa reflexión en tanto hay reconocimiento del problema de bajo desempeño académico y prevalece el deseo de superarlo, se siente el esfuerzo de su familia como una motivación para hacerlo, pues consideran importante retribuir el trabajo realizado por los padres para apoyar su formación académica.

Por otro lado se observaron narrativas en los estudiantes que dan cuenta de la importancia de la familia como un apoyo, no solo económico, sino también emocional. Se valora nuevamente el esfuerzo de los padres como un elemento motivador que se responde con buenos resultados académicos. Tal como se expresa a continuación:

(...) uno no tiene como ese criterio de decir, no es que a mi papá le duele, le toca trabajar para darme la plata, entonces uno no valora, porque uno piensa, sé que el otro semestre me va a pagar, sé que el otro semestre voy a estar estudiando, entonces eso también es muy frustrante, a veces porque uno reacciona y uno se da cuenta de que los papás no, o sea no la tienen ahí guardada y ellos tienen que pasar muchas cosas para que nosotros estemos acá. (P1, Esc. 2)

Finalmente se reconoce que se presentan recursos en los estudiantes a partir de la relación y 
representación que se tiene de la familia, sin embargo no se logran presentar acontecimientos, entendidos como las experiencias de cambio de los estudiantes, esto permite pensar que es importante continuar con los escenarios de vinculación de la familia al entorno académico, que permita continuar fortaleciendo el crecimiento y desarrollo profesional de los estudiantes.

\section{Memorias - institución}

En cuanto a las memorias que surgen, se reconoce que los estudiantes han logrado encontrar en las tutorías un elemento importante que les permite pensarse distinto en cuanto a su situación de prueba académica. Así mismo, los estudiantes comienzan a reconocer la responsabilidad que tienen en la inasistencia a clase y como esta tiene consecuencias negativas en su desempeño.

E3: Pues mi tutora me parece una persona (...) y pues este semestre sí ya, ya ha habido una mejor comunicación porque ella me dice que está pendiente de mis notas y que ella misma se da cuenta de (...) ahí vamos bien (...) (Esc.1)

En este sentido, los estudiantes dan cuenta de la corresponsabilidad en el proceso enseñanza y aprendizaje. Algunos estudiantes consideran pertinente abrir diálogos entre las partes que permitan la retroalimentación de los roles en la relación, pese a que en otros relatos aparece un comportamiento pasivo en este aspecto y la explicación de los estudiantes la relacionan con la no apertura de algunos profesores y por sentimientos de temor a la represaría al expresar algo que genere desacuerdo en el docente.

\section{P3. "Es miedo al docente" (Esc.2)}

De igual forma para la movilización de los recursos universitarios, el estudiante reconoce la necesidad de realizar un diagnóstico que favorezca la comprensión del problema. También se observa el planteamiento de estrategias personales en pro de la relación con el docente como un cambio de disposición, actitud y mejor uso del tiempo libre.
E3: Bueno, en mi caso hay una materia que no he entendido, y casi como desde la segunda semana que entramos a clases me he acercado al profesor para que me dé tutoriales. (Esc.1)

Dentro de las narrativas analizadas de los escenarios no se identificó ninguna que pudiera ser clasificada como "acontecimiento", lo cual puede comprenderse como la necesidad de un trabajo interventivo que permita la movilización de las narrativas alternas identificadas hacia la resignificación en los estudiantes de lograr un rol activo y autónomo, en pro de su desempeño académico, con la proposición e implementación de estrategias para mejorar en los diferentes aspectos que ellos consideran, tienen que ver con mejoramiento y cambio de su situación problema.

\section{Discusión}

\section{Factor psicosocial}

La presente investigación, como bien lo plantea Jackson, Sher y Cooper (2003), asumen que el desempeño académico es un fenómeno multifactorial, que tiene en cuenta componentes psicológicos y sociales, entre los que se encuentran la edad, la autoestima, el nivel socioeconómico, el lugar de residencia y características familiares, como el nivel de escolaridad y ocupación de los padres y la calidad del ambiente que rodea al estudiante, entre otras variables, como se describirá a lo largo de los análisis presentados.

De acuerdo con lo anterior, la delimitación del factor psicosocial pretende tener en cuenta elementos de lo personal y social, presentes en la vida de los estudiantes que hicieron parte de la presente investigación, al encontrar que en el apartado de lo personal prevalece el reconocimiento de tópicos como la administración del tiempo, la asistencia a clases y la búsqueda de recurso externos, como asesorías, búsqueda de ayuda, grupos de estudio, están a favor de tener mejores resultados académicos.

Como lo menciona Montero, Villalobos y Valverde (2007), los factores psicosociales se consideran 
como las conexiones que se dan entre las personas y la sociedad, pues es evidente que ejercen una clara influencia sobre hombres y mujeres. En esta dimensión se incluyen variables que miden ciertos rasgos de personalidad que podrían estar asociados al desempeño, tales como la motivación, la ansiedad, la autoestima en contextos académicos y la percepción que el estudiante tiene del "clima académico", considerando el conocimiento y el grado de entusiasmo que percibe de los profesores, lo cual coincide con los hallazgos de la presente investigación, donde se ponen en juego características personales en interacción con el factor institucional, el cual tiene todo que ver con el contexto universitario.

Otro elemento importante es cuando los estudiantes en prueba académica afirman que una de las consecuencias que perciben con mayor fuerza es la de atrasarse y hacen comparaciones con los pares que van en línea. En consecuencia, la autoestima suele declinar. No obstante empieza a aumentar hacia la mitad y el final de la adolescencia, a medida que los jóvenes van adquiriendo más habilidades y responsabilidades adultas. La autoestima está vinculada con la competencia real de los adolescentes en ámbitos que son importantes para ellos y también tiene relación con la manera en que son percibidos por sus padres y amigos (Kail y Cavanaugh 2011).

Los hallazgos de la investigación permiten dar cuenta de la relación existente entre la motivación y el desempeño, en la medida en que el estudiante muestra más interés por lo que realiza y sus aspiraciones se ajustan a sus posibilidades, gana un mayor nivel de compromiso y motivación, porque logra conectar con sentido su quehacer en la universidad con su proyecto de vida.

También se encontró que los estudiantes universitarios logran el reconocimiento de sus recursos, tanto personales, como intelectuales y sociales. Sin embargo se observa especialmente en los que tienen bajo desempeño académico, dificultades para ponerlos al servicio de la construcción de estrategias que les permitan mejorar su situación, lo cual se relaciona con las estrategias evitativo pasivas, lo que mantiene el bajo desempeño académico.
A partir de lo anterior, se evidencia que el desempeño académico de los estudiantes se ve afectado por sus niveles de ansiedad y otras características personales que podrían operar como facilitadores o inhibidores del desempeño.

\section{Factor familia}

Luego de realizar la revisión de los resultados cualitativos se identificó la importancia de la familia en la vida de los estudiantes, de tal manera que esto puede operar como un apoyo y una motivación para su proyecto universitario, encontrándose también una relación marcada entre mayor percepción de apoyo familiar y mejor desempeño académico. Así como también los problemas familiares pueden configurarse como un factor que interfiere en dicho desempeño, lo que lleva al estudiante a considerar como estrategia poner límites a sus diferentes situaciones de vida, como bien lo expresa Galacia, Sánchez y Robles (2013), al partir de la trascendencia que posee la familia en generar responsabilidad en el joven por medio de desarrollo de actitudes comunicativas asertivas y trabajo en equipo, o desde una perspectiva de competitividad y hostilidad social.

Así mismo, en esta investigación se identificó que los estudiantes logran valorar el esfuerzo de los padres, aspecto que se configura como un agente de motivación para corresponder con buenos resultados académicos. También se dejan ver las razones por las cuales los estudiantes, a pesar de contar con los recursos intelectuales, entienden su bajo desempeño académico en relación con actitudes como la pereza, manejo inadecuado del tiempo, bajo interés por lo académico, y prefieren realizar actividades como dormir, salir con los amigos, ver televisión, entre otras.

Esto se relaciona con lo planteado por González et al. (2003), quien afirma que el joven se centra en esta dimensión sin controlar la distribución de sus responsabilidades, para lo cual es vital que los padres desde la infancia regulen estos comportamientos, a tal fin que su incidencia no sea opuesta al fin que se quisiera lograr, con adecuadas pautas de crianza y detección o manejo a tiempo por orientación de profesionales. De igual forma 
Robledo y García (2009), plantean que el ambiente familiar, el funcionamiento y la estructura y/o clima son otros de los factores clave para el desarrollo integral y escolar del niño, lo que impacta su futuro académico.

También se observó que algunas familias influyen en la elección de la carrera, comentan que en ocasiones la familia tiene una tradición en la formación y se espera que ellos den continuidad a esta línea. Esto se logra articular con lo planteado por Guevara, Jaramillo y Tovar (2013); quienes lograron identificar que existen implicaciones entre el nivel educativo del padre y las expectativas del estudiante, lo que puede entenderse si se considera que en la estructuración de la familia y el manejo de autoridad y roles, se encuentra que un gran porcentaje vive en familias donde existe claridad en estos aspectos, por lo que la figura paterna mantiene un significado y representatividad importante en el sistema familiar.

Cabe entonces considerar a la familia como un sistema, en el cual los subsistemas interactúan afectándose entre sí, esa afectación no depende de la estructura, es decir, se dará independientemente de si la familia es de tipo monoparental, nuclear, compuesta, extensa o reorganizada. Esto de igual forma se relaciona con lo planteado por Montero et al. (2007), al reconocer que la motivación y la autoestima están directamente implicadas en el rendimiento, siendo un escenario posibilitador el pensarse que existe un proceso de responsabilidad compartida y organizando así un papel más activo de los estudiantes.

Así mismo se evidencia dentro de los relatos que se presentan, procesos de reflexión, en tanto que hay reconocimiento del problema de bajo desempeño académico y el deseo de superarlo, destacando el esfuerzo de su familia como un elemento motivador, pues consideran importante retribuir el trabajo realizado por los padres para apoyar su formación académica. Por tanto es necesario que se visualicen estrategias de cambio que les permita posicionarse desde un discurso con mayor fuerza, que dé cuenta de la posibilidad de empezar a agenciar cambios. Esto se relaciona con lo planteado por Torres y Rodríguez (2006), que, al no satisfacerse, pueden conducir a decepciones tempranas $y$, por consiguiente, se convierte en un factor de deserción y cuando el estudiante no logra un adecuado rendimiento académico en las asignaturas del plan de estudios y la institución no le proporciona las herramientas necesarias para superar las deficiencias académicas. En el presente estudio se muestra una relación entre el contexto familiar y el rendimiento académico, esta relación no es lineal, pero se puede inferir que esos dos ambientes de desarrollo del estudiante están relacionados, y que intervenir en el contexto familiar puede llevar a que los estudiantes universitarios alcancen logros académicos evidentes.

\section{Factor institucional}

En la presente investigación se identificó la relación del factor institucional con el desempeño académico, entendido este factor como todo lo que engloba el contexto de la Universidad de Boyacá como políticas institucionales, modelo pedagógico, relación docente estudiante, infraestructura que recoge tanto los recursos físicos como los humanos, entre otras variables.

Un elemento que se presenta con relevancia es la relación entre docente y estudiante, puesto que se considera que la rigidez de la relación dificulta un buen desempeño. Así mismo, la convivencia dentro de la clase se configura como un factor fundamental para mejorar o dificultar el desempeño, debido a que en ocasiones el estudiante considera que la rigidez del docente le inhibe la búsqueda de ayuda. Esto se puede relacionar con lo planteado por Saccsa (2010), quien reconoce el impacto del clima institucional en el rendimiento académico de los docentes y esto a su vez se relaciona con las estrategias pedagógicas y la posibilidad de diversificar los contextos de desarrollo de eventos culturales y actividades extracurriculares que le permitan otros espacios.

En el mismo sentido Montero et al. (2007) reconocen que la motivación, la ansiedad, la autoestima en contextos académicos y la percepción que el estudiante tiene del "clima académico", está influenciado por el conocimiento y el grado de entusiasmo que percibe del profesor. Es pues el 
significado que se le atribuye al docente por parte del estudiante, un elemento que organiza este "clima académico", lo que genera preocupación en tanto la narrativa hacia el docente es de orden deficitario.

Por otro lado, los estudiantes organizan un reconocimiento desde la responsabilidad compartida con el docente en el proceso de enseñanza y aprendizaje, así mismo resaltan la importancia de su papel como guía y orientador al realizar procesos de retroalimentación continúa.

Lo anterior también coincide con lo planteado por Duart y Sangra (2000), quienes señalan que el éxito del rendimiento académico del estudiante depende de la eficiencia con la que cuente el profesor y de su capacidad para motivarlo, al atender las diferentes necesidades identificadas en las clases. El resultado de estas acciones necesariamente influirá en los resultados del desempeño académico de los estudiantes.

Para finalizar la discusión se puede decir que la presente investigación permitió un acercamiento comprensivo a la complejidad presente en el desempeño académico de los estudiantes de la Universidad de Boyacá, sede Sogamoso, en relación con los diferentes factores familiares, psicosociales e institucionales, que participan en el mantenimiento o cambio en el fenómeno estudiado. Como bien lo plantea Garbanzo y Guillese (2007), el rendimiento académico por ser multicausal se envuelve en una enorme capacidad explicativa de los distintos factores y espacios temporales que intervienen en el proceso de aprendizaje.

\section{Referencias}

Berger, S. et al. (2013). Rendimiento académico y las dimensiones personal y contextual del aprendizaje socioemocional: evidencias de su asociación en estudiantes chilenos. Universitas Psychologica, 13(2), 627-638.

Chica, S., Galvis, D., \& Ramírez, A. (2009). Determinantes del rendimiento académico en Colombia. Pruebas ICFES - Saber 11o, 2009. Revista Universidad EAFIT, 46(160), 120-136.
Duart, J. \& Sangra, A. (2000). Aprender en la virtualidad. Catalunya, España: Gedisa.

Echeverría, R. (2003). Ontología del lenguaje. Chile: Nordesde Ltda.

Galacia, M., Sánchez, A., \& Robles, F. (2013). Autoeficacia en escolares adolescentes: su relación con la depresión, el rendimiento académico y las relaciones familiares. Revista Anales de Psicología, 29(2), 491-500.

Garbanzo, V. \& Guiselle, M. (2007). Factores asociados al rendimiento académico en estudiantes universitarios, una reflexión desde la calidad de la educación superior pública. Revista Educación, 31(1), 43-63.

González, J., Núñez, J., Álvarez, L., González, S., González, P., ... Bernardo, A. (2003). Adaptabilidad y cohesión familiar, Implicación parental. Psicothema, 15(3), 471-477.

Guevara, E., Jaramillo, R., \& Tovar, S. (2013). Factores familiares y su relación con el rendimiento académico en estudiantes de Psicología. Revista Virtual Universidad Católica del Norte, 5(4), 122-140.

Guzmán, R. \& Pacheco, M. (2014). Comunicación familiar y desempeño académico en estudiantes universitarios. Revista del Instituto de Estudios en Educación Universidad del Norte. 1(20) 79-91.

Jackson, K. M., Sher, K. J., \& Cooper, M. L. (2002). Adolescent alcohol and tobacco use: onset, persistence and trajectories of use across two samples. Addiction, 97(5) 517-531.

Jornet, J. (2012), Las dimensiones docentes y cohesión social: Reflexiones desde la evaluación. Revista Iberoamericana de Evaluación Educativa, 5(1) 349-400.

Kail, R. \& Cavanaugh, J. (2011). Desarrollo humano. Una perspectiva de ciclo vital (5a edición). México: Ed. Cengage Learning. 
Luthar, S. \& Blatt, S. J. (1995). Differential vulnerability of dependency and self-criticism among disadvantaged teenagers. Journal of Research on Adolescence, 5(4), 431-449.

Minuchin, S. \& Fishman, C. (2004). Técnicas de terapia familiar. ( $1^{a}$ edición). Buenos Aires: Paidós.

Montero, E., Villalobos, J., \& Valverde, A. (2007). Factores institucionales, pedagógicos, psicosociales y sociodemográficos asociados al rendimiento académico en la Universidad de Costa Rica: Un análisis multinivel. RELIEVE. Revista electrónica de Investigación y Evaluación Educativa, 13(2), 215-234.

Montes, I. \& Lerner, J. (2011). Rendimiento académico de los estudiantes de pregrado de la Universidad EAFIT. Medellín: Universidad EAFIT, grupo de estudios en economía y empresa. Recuperado de http://www.eafit.edu. co/institucional/calidadeafit/investigacion/ Documents/Rendimient\%20Ac\%C3\%A1demicoperrspectiva\%20cuantitativa.pdf

Pérez, M. \& Urquijo, S. (2001). Depresión en adolescentes. Relaciones con el desempeño académico. Psicología escolar e educacional, 5(1), 49-58.

Reinherz, H. Z., Frost, A. K., \& Pakiz, B. (1991). Changing faces: correlates of depressive symptoms in late adolescence. Family and Community Health, 14(3), 52-63.

Reparaz, C., Touron, J., \& Villanueva, C. (1990). Estudio de algunos factores relacionados con el rendimiento académico en $8^{\circ}$ de EGB. Revista de Pedagogía Bordón, 42(2), 501-527.

Robledo, P. \& García, J. (2009). El entorno familiar y su influencia en el rendimiento académico de los alumnos con dificultades de aprendizaje: Revisión de estudios empíricos. Universidad de Oviedo ICE, 37(1), 117-128.

Saccsa, J. (2010). Relación entre clima institucional y el desempeño académico de los docentes de los Centros de Educación Básica Alternativa (CEBAS) del Distrito de San Martín de Porres (Tesis de Maestría). Universidad Nacional Mayor San Marcos, Lima, Perú.

Soares, A., Almeida, L. \& Guisande, A. (2011). Ambiente académico y adaptación a la universidad: un estudio con estudiantes de $1^{\circ}$ año de la Universidad Do Minho. Revista Iberoamericana de Psicología y Salud, 2(1), 99-121.

Torres, L. \& Rodríguez, N. (2006). Rendimiento académico y contexto familiar en estudiantes universitarios. Enseñanza e Investigación en Psicología. Universidad Veracruzana, 11(002), 255-270. 